OPEN ACCESS

Edited by:

Mirza Hasanuzzaman, Sher-e-Bangla Agricultural University,

Bangladesh

Reviewed by:

Sergey Shabala

University of Tasmania, Australia

Kamrun Nahar

Kagawa University, Japan

${ }^{*}$ Correspondence:

Ewa Niewiadomska e.niewiadomska@ifr-pan.edu.pl

Specialty section: This article was submitted to Plant Physiology,

a section of the journal

Frontiers in Plant Science

Received: 30 April 2016 Accepted: 22 July 2016 Published: 04 August 2016

Citation:

Pilarska M, Wiciarz M, Jaiić $I$, Kozieradzka-Kiszkurno M, Dobrev P, Vanková $R$ and Niewiadomska $E$

(2016) A Different Pattern

of Production and Scavenging

of Reactive Oxygen Species in Halophytic Eutrema salsugineum (Thellungiella salsuginea) Plants in Comparison to Arabidopsis thaliana and Its Relation to Salt Stress

Signaling. Front. Plant Sci. 7:1179. doi: 10.3389/fp/s.2016.01179

\section{A Different Pattern of Production and Scavenging of Reactive Oxygen Species in Halophytic Eutrema salsugineum (Thellungiella salsuginea) Plants in Comparison to Arabidopsis thaliana and Its Relation to Salt Stress Signaling}

\author{
Maria Pilarska', Monika Wiciarz², Ivan Jajić2, Małgorzata Kozieradzka-Kiszkurno ${ }^{3}$, \\ Petre Dobrev', Radomíra Vanková ${ }^{4}$ and Ewa Niewiadomska ${ }^{1 *}$
}

\begin{abstract}
'The Franciszek Górski Institute of Plant Physiology - Polish Academy of Sciences, Kraków, Poland, ${ }^{2}$ Department of Plant Physiology and Biochemistry, Faculty of Biochemistry Biophysics and Biotechnology, Jagiellonian University, Kraków, Poland, ${ }^{3}$ Department of Plant Cytology and Embryology, University of Gdańsk, Gdańsk, Poland, ${ }^{4}$ Institute of Experimental Botany AS CR, Prague, Czech Republic
\end{abstract}

Isolated thylakoids from halophytic Eutrema salsugineum (Thellungiella salsuginea) produces more $\mathrm{H}_{2} \mathrm{O}_{2}$ in comparison to glycophytic Arabidopsis thaliana. The first objective of this study was to verify whether this feature is relevant also to the intact chloroplasts and leaves. Enhanced $\mathrm{H}_{2} \mathrm{O}_{2}$ levels in chloroplasts and leaves of E. salsugineum were positively verified with several methods (electron microscopy, staining with Amplex Red and with diaminobenzidine). This effect was associated with a decreased ratio of $\mathrm{O}_{2}{ }^{-}-\mathrm{H}_{2} \mathrm{O}_{2}$ in $E$. salsugineum in comparison to $A$. thaliana as detected by electron paramagnetic resonance method. As a next step, we tested how this specific ROS signature of halophytic species affects the antioxidant status and down-stream components of ROS signaling. Comparison of enzymatic antioxidants revealed a decreased activity of ascorbate peroxidase (APX), enhanced activity of glutathione peroxidase, and the presence of thylakoid-bound forms of iron superoxide dismutase (FeSOD) and APX in E. salsugineum. These cues were, however, independent from application of salt stress. The typical $\mathrm{H}_{2} \mathrm{O}_{2}$-dependent cellular responses, namely the levels of glucosinolates and stress-related hormones were determined. The total glucosinolate content in E. salsugineum water-treated leaves was higher than in A. thaliana and increased after salinity treatment. Treatment with salinity up-regulated all of tested stress hormones, their precursors and catabolites [abscisic acid (ABA), dihydrophaseic acid, phaseic acid, 1-aminocyclopropane-1-carboxylic acid, salicylic acid, jasmonic acid, cis-(+)-12-oxo-phytodienoic acid and jasmonoyl-L-isoleucine] in A. thaliana, whereas in E. salsugineum only a stimulation in ethylene synthesis and ABA catabolism was noted. Obtained results suggest that constitutively enhanced $\mathrm{H}_{2} \mathrm{O}_{2}$ 
generation in chloroplasts of $E$. salsugineum might be a crucial component of stressprepardeness of this halophytic species. It shapes a very efficient antioxidant protection (in which glucosinolates might play a specific role) and a fine tuning of hormonal signaling to suppress the cell death program directed by jasmonate pathway.

Keywords: chloroplast, glucosinolates, halophyte, hydrogen peroxide, salinity, stress hormones

\section{INTRODUCTION}

Reactive oxygen species (ROS) are intriguing molecules, which are toxic to the biological structures but also play a signaling role in controlling plant growth, development and stress responses (for a recent review, see Suzuki et al., 2012; Baxter et al., 2014). They accompany the basal metabolic fluxes of aerobic organisms during the whole ontogeny. Considering the precise and multilevel control of metabolic changes it might not be surprising that ROS coming from the various cellular and extracellular sites seem to have their specific signaling targets (op den Camp et al., 2003; Avsian-Kretchmer et al., 2004; Gadjev et al., 2006; Geisler et al., 2006; Laloi et al., 2007). In plants several ROS, such as $\mathrm{H}_{2} \mathrm{O}_{2}, \mathrm{O}_{2}{ }^{-}-$and ${ }^{1} \mathrm{O}_{2}$, are formed in chloroplasts aside the photosynthetic electron transport. They, in turn, affect the nuclear gene expression to adjust the photosynthesis to changing environment. The most stable form, which is assumed to leave this organelle and evoke the effects on the nuclear genes, is $\mathrm{H}_{2} \mathrm{O}_{2}$ (Mubarakshina et al., 2010; Borisova et al., 2012).

In an attempt to recognize the signaling effects of $\mathrm{H}_{2} \mathrm{O}_{2}$ originating from chloroplasts, Arabidopsis mutant overexpressing glycolate oxidase in chloroplasts (GO5 plants) has been developed (Fahnenstich et al., 2008). In the photorespiratory conditions $\mathrm{GO} 5$ mutants produce $\mathrm{H}_{2} \mathrm{O}_{2}$ in chloroplasts instead of in peroxisomes. Transcript profiling of GO5 plants (Balazadeh et al., 2012) identified the $\mathrm{H}_{2} \mathrm{O}_{2}$ regulated genes and transcription factors, whereas further work of Sewelam et al. (2014) underpinned the top 20 genes specifically up-regulated by $\mathrm{H}_{2} \mathrm{O}_{2}$ produced in chloroplasts. These studies proved that $\mathrm{H}_{2} \mathrm{O}_{2}$ can trigger different responses depending on the subcellular site of its production.

Recently, we demonstrated that thylakoids isolated from a highly stress resistant species Eutrema salsugineum (Thellungiella salsuginea) are capable of the enhanced production of $\mathrm{H}_{2} \mathrm{O}_{2}$, in comparison to Arabidopsis thaliana, already in the absence of stress (Wiciarz et al., 2015). This creates the opportunity to unravel the signaling action of chloroplast $\mathrm{H}_{2} \mathrm{O}_{2}$ generation in the natural system. E. salsugineum tolerates extreme salinity, cold, drought, ozone and over the last years this species became a plant model of stress resistance well-comparable with the Arabidopsis genome (Inan et al., 2004; Li et al., 2006; Amtmann, 2009; Hou and Bartels, 2015). So far, several studies focused on the discovery of transcriptomic footprints of high stress resistance in E. salsugineum. These studies showed that several stress-associated genes in E. salsugineum have a constitutively higher expression in comparison with $A$. thaliana already in the absence of stress (Inan et al., 2004; Taji et al., 2004; Gong et al., 2005). The group of up-regulated genes includes, for example, those involved in abscisic acid ( $A B A$ ) biosynthesis and signaling (Taji et al., 2004; Gong et al., 2005). In contrast, after stress treatment, only a slight change in gene expression was detected in E. salsugineum in comparison with a great activation of transcription in $A$. thaliana plants (Taji et al., 2004; Li et al., 2006; Wong et al., 2006). Also, a comparative proteomics of $A$. thaliana and E. salsugineum salt responses revealed more changes in protein abundance in Arabidopsis than in Eutrema (Pang et al., 2010). Combined, these results indicate so called 'stress preparedness' of E. salsugineum, which supports its halophytic nature. Comparison of metabolite profiles of these two species following salt stress revealed a significant differences (Arbona et al., 2010). This suggests, that an adjustment of metabolism and activation of the already present enzymatic machinery serves as a faster and more efficient strategy to cope with stress than synthesis of new proteins.

A goal of this study was to verify whether enhanced generation of $\mathrm{H}_{2} \mathrm{O}_{2}$ in thylakoids of halophytic Eutrema in comparison to glycophytic $A$. thaliana is significant also in vivo. As a next objective, it was tested how this situation influences oxidative damage, antioxidant system and hormonal signaling in the control conditions and after a salinity stress.

\section{MATERIALS AND METHODS}

\section{Plant Material and Growth Conditions}

Arabidopsis thaliana (Col-0) and E. salsugineum (T. salsuginea, salt cress) ecotype Shandong were grown from seeds in the soil culture under irrigation with tap water. Seeds were obtained from the Nottingham Arabidopsis Stock Centre, UK. Plants were cultivated in the phytotron chamber at temperatures of $18^{\circ} / 16^{\circ} \mathrm{C}$ day/night, photoperiod $10 / 14 \mathrm{~h}$, irradiance of $\mathrm{ab}$. $220 \mu \mathrm{mol} \mathrm{m}^{-2} \mathrm{~s}^{-1}$ and $\mathrm{RH} \sim 50 \%$. Both species were adapted to these conditions for at least three generations. Because of delay in growth of E. salsugineum in comparison to A. thaliana, as reported earlier (Inan et al., 2004; Stepien and Johnson, 2009), to compare rosettes at the same developmental stage, 4-weeksold A. thaliana and 5-weeks-old E. salsugineum plants were taken for experiments. To evoke a mild salinity-stress plants were irrigated with 0.15 and $0.3 \mathrm{M} \mathrm{NaCl}$ solutions for A. thaliana and $E$. salsugineum, respectively, while watered plants served as controls. After 7 days of $\mathrm{NaCl}$ treatment complete rosettes were collected, frozen in liquid nitrogen and stored at $-80^{\circ} \mathrm{C}$ until further use (unless stated otherwise).

\section{Thylakoid Membrane Preparation}

Thylakoids were isolated as described earlier (Wiciarz et al., 2015). Shortly, leaves were homogenized in medium containing 50 mM HEPES-KOH (pH 7.6), 330 mM sorbitol (control plants) 
and $495 \mathrm{mM}$ sorbitol (NaCl-treated plants) respectively, $1 \mathrm{mM}$ $\mathrm{MgCl}_{2}, 2 \mathrm{mM} \mathrm{Na}$ EDTA, $5 \mathrm{mM}$ sodium ascorbate and $0.01 \%$ $(\mathrm{w} / \mathrm{v})$ fatty acid-free bovine serum albumine. After centrifugation for $4 \mathrm{~min}$ at $4000 \times g$ the pellet was resuspended in $50 \mathrm{mM}$ HEPES-KOH (pH 7.6), $5 \mathrm{mM}$ sorbitol, $5 \mathrm{mM} \mathrm{MgCl}_{2}$ and centrifuged again. Then the pellet was washed and resuspended in $50 \mathrm{mM}$ HEPES-KOH ( $\mathrm{pH} 7.6$ ), $330 \mathrm{mM}$ sorbitol, $10 \mathrm{mM}$ $\mathrm{MgCl}_{2}, 20 \mathrm{mM} \mathrm{NaCl}, 2.5 \mathrm{mM} \mathrm{Na} \mathrm{mDTA}_{2} 10 \mathrm{mM} \mathrm{NaHCO}$. The chlorophyll concentration was estimated spectrophotometrically according to Lichtenthaler and Buschmann (2001).

\section{Electron Paramagnetic Resonance (EPR) Measurements}

Production of $\mathrm{O}_{2}^{\bullet-}$ and $\mathrm{H}_{2} \mathrm{O}_{2}$ by thylakoids from $A$. thaliana and $E$. salsugineum water-treated plants was detected by electron paramagnetic resonance (EPR) spin-trapping spectroscopy using DMPO (5,5-dimethyl-pyrroline $N$-oxide; Sigma-Aldrich, USA) and POBN [ $\alpha$-(4-pyridyl-1-oxide)- $N$-tertbutylnitrone; SigmaAldrich] as the spin trap, respectively, as described earlier (Jajić et al., 2015). Shortly, for $\mathrm{O}_{2}^{\bullet}-$ detection, isolated thylakoids (chlorophyll concentration $200 \mu \mathrm{g} \mathrm{mL}^{-1}$ ) were mixed with DMPO to a final concentration $50 \mathrm{mM}$, transferred to a flat cell and illuminated for $5 \mathrm{~min}$ at $500 \mu \mathrm{mol}$ quanta $\mathrm{m}^{-2} \mathrm{~s}^{-1}$ within the EPR spectrometer MiniScope MS300 (Magnettech $\mathrm{GmbH}$, Germany). For $\mathrm{H}_{2} \mathrm{O}_{2}$ detection, the $\mathrm{H}_{2} \mathrm{O}_{2}$-derived hydroxyl radical after the initiation of the Fenton reaction was measured. Isolated thylakoids (concentration of chlorophyll $150 \mu \mathrm{g} \mathrm{mL}^{-1}$ ) in a reaction medium $\mathrm{pH} 7.6$ containing $0.4 \mathrm{M}$ sucrose, $20 \mathrm{mM}$ $\mathrm{NaCl}, 5 \mathrm{mM} \mathrm{MgCl}, 10 \mathrm{mM}$ Hepes-KOH were illuminated with white light source LS2 (Hansatech, UK) for $3 \mathrm{~min}$ at $500 \mu \mathrm{mol}$ quanta $\mathrm{m}^{-2} \mathrm{~s}^{-1}$. Next, $50 \mathrm{mM}$ POBN, $50 \mu \mathrm{M}$ FeEDTA and $4 \%$ ethanol were added and incubated for $3 \mathrm{~min}$, transferred to glass capillaries and measured using an EPR spectrometer. To check the influence of enzymatic scavengers on $\mathrm{H}_{2} \mathrm{O}_{2}$ production, measurements were performed in presence and absence of $5 \mathrm{mM}$ sodium azide (inhibitor of heme containing enzymes).

\section{$\mathrm{H}_{2} \mathrm{O}_{2}$ Detection}

Ultrastructural localization of $\mathrm{H}_{2} \mathrm{O}_{2}$ was visualized in transmission electron microscopy (TEM) via electron-dense precipitates of cerium perhydroxides $\left[\mathrm{Ce}(\mathrm{OH})_{2} \mathrm{OOH}\right.$ and $\left.\mathrm{Ce}(\mathrm{OH})_{3} \mathrm{OOH}\right]$ formed after the reaction of cerium chloride $\left(\mathrm{CeCl}_{3}\right)$ with endogenous $\mathrm{H}_{2} \mathrm{O}_{2}$ (Bestwick et al., 1997). After $1 \mathrm{~h}$ of light leaves of $A$. thaliana and E. salsugineum from water-treated plants were cut into small pieces $(\sim 5 \mathrm{~mm})$. Leaf fragments were immediately infiltrated and then incubated for $1 \mathrm{~h}$ with a $0.5 \mathrm{M}$ morpholinepropanesulfonic acid (MOPS) buffer ( $\mathrm{pH}$ 7.0), containing $5 \mathrm{mM} \mathrm{CeCl}_{3}$ (Libik-Konieczny et al., 2015). The controls were tissue samples incubated in MOPS buffer. Then, tissues were quickly washed in the 0.5 M MOPS and fixed in $2.5 \%(\mathrm{w} / \mathrm{v})$ formaldehyde (prepared from paraformaldehyde) and a $2.5 \%(\mathrm{v} / \mathrm{v})$ glutaraldehyde in $50 \mathrm{mM}$ cacodylate buffer $(\mathrm{pH}$ 7.0) for $4 \mathrm{~h}$ at room temperature. The procedure for preparing the samples for TEM was as described earlier (KozieradzkaKiszkurno and Płachno, 2012). The material was dehydrated in a series of graded acetone and embedded in Spurr Low-Viscosity Embedding Kit (Polysciences, Germany). Ultrathin (60-90 nm) sections were cut with a diamond knife on a Leica EM UC7 ultramicrotome. The sections were stained with uranyl acetate and lead citrate and then viewed using a Philips CM 100 TEM at $75 \mathrm{kV}$.

Histochemical localization of $\mathrm{H}_{2} \mathrm{O}_{2}$ production in leaves was determined using the DAB (3,3-diaminobenzidine) staining technique according to Libik-Konieczny et al. (2015). Leaves of $E$. salsugineum and $A$. thaliana water-treated plants were infiltrated with a solution of $1 \mathrm{mg} \mathrm{mL}{ }^{-1} \mathrm{DAB}$ (Sigma-Aldrich) prepared in water. Incubation was carried for $4 \mathrm{~h}$ in the dark at room temperature.

\section{SDS-PAGE and Immunoblot Analysis}

Leaf soluble proteins were extracted with $0.1 \mathrm{M}$ phosphate buffer pH 7.5 containing $1 \mathrm{mM}$ dithiothreitol, 2\% (w/v) polyvinylpolypyrrolidone and protease inhibitor cocktail (SigmaAldrich). Homogenates were centrifuged $10 \mathrm{~min}$ at $10000 \times g$. Protein concentration in supernatant was estimated using Roti ${ }^{\circledR}$ Nanoquant Protein quantitation assay (Carl Roth, Germany). SDS-PAGE as well as immunoblotting were performed as described earlier (Niewiadomska et al., 2009). Thylakoid membranes (TMs) and soluble proteins were dissolved in denaturating buffer and heated $20 \mathrm{~min}$ at $99^{\circ} \mathrm{C}$ (soluble proteins) or $5 \mathrm{~min}$ at $75^{\circ} \mathrm{C}$ (TMs). After electrophoresis, the separated proteins were blotted onto nitrocellulose membranes and probed with polyclonal antibodies raised against: ascorbate peroxidase (APX; anti-APX); glutathione peroxidase (GPX; antiGPX); iron superoxide dismutase (FeSOD; anti-FeSOD); gamma glutamylcysteine synthase, $\gamma$-ECS (anti- $\gamma$-ECS); peroxiredoxins Q, PrxQ (anti-PrxQ). All antibodies were purchased from Agrisera (Sweden).

\section{Determination of Lipid Peroxidation}

Lipid peroxidation in leaves and isolated thylakoids of $A$. thaliana and E. salsugineum water-treated plants was assessed by measuring the malondialdehyde (MDA) content using highperformance liquid chromatography (HPLC) as described by Rastogi et al. (2014). Shortly, leaf samples were ground in a mortar with chilled $80 \%(\mathrm{v} / \mathrm{v})$ ethanol. The extract was then centrifuged $(10000 \times g$ for $2 \mathrm{~min})$ and the supernatant was further used. Samples were mixed with equal amount of reaction mixture containing 20\% (w/v) trichloroacetic acid, $0.01 \%(\mathrm{w} / \mathrm{v})$ butylated hydroxytoluene and $0.65 \%(\mathrm{v} / \mathrm{v})$ thiobarbituric acid (TBA). After heating at $95^{\circ} \mathrm{C}$ for $20 \mathrm{~min}$ and centrifugation the MDA-(TBA $)_{2}$ adduct was separated and quantified by the HPLC. The elution buffer was $50 \mathrm{mM} \mathrm{KH} \mathrm{PO}_{4}$ ( $\left.\mathrm{pH} 7.0\right) /$ methanol $(65: 35, \mathrm{v} / \mathrm{v})$. The retention time was $5 \mathrm{~min}$ and flow rate of $0.5 \mathrm{~mL} \mathrm{m^{-1 }}$ with detection at $530 \mathrm{~nm}$. Tetraethoxy-propane (Sigma-Aldrich) was used as a standard.

\section{Quantification of $\mathrm{H}_{2} \mathrm{O}_{2}$ Concentration}

An Amplex ${ }^{\circledR}$ Red Hydrogen Peroxide/Peroxidase Assay Kit (Life Technologies/Thermo Fisher Scientific, USA) was used to measure $\mathrm{H}_{2} \mathrm{O}_{2}$ content in $A$. thaliana and $E$. salsugineum leaves after water irrigation. Leaves $(0.1 \mathrm{~g})$ frozen in liquid 
nitrogen were ground with $0.5 \mathrm{M}$ sodium phosphate buffer, $\mathrm{pH} 7.4$, and centrifuged $10 \mathrm{~min}$ at $10,000 \times \mathrm{g}$. Proteins were removed from the extracts using Amicon Ultra Centrifugal Filters (Merck Millipore, USA) and the filtrate $(25 \mu \mathrm{L})$ was incubated for $30 \mathrm{~min}$ in dark conditions with $50 \mathrm{mM}$ Amplex Red reagent and 0.2 units $\mathrm{mL}^{-1}$ horseradish peroxidase. Excitation was measured at $530 \mathrm{~nm}$ and fluorescence detection at $590 \mathrm{~nm}$ with a microplate reader Synergy 2 (BioTek, USA). The hydrogen peroxide concentration was estimated by comparison with standard curve $(0-10 \mu \mathrm{M})$ of $\mathrm{H}_{2} \mathrm{O}_{2}$. The experiments were repeated three times independently, each time in triplicate.

\section{Determination of Antioxidant Enzyme Activities}

Soluble leaf proteins were extracted with $0.1 \mathrm{M}$ phosphate buffer $\mathrm{pH} 7.5$ [catalase (CAT); SOD], $50 \mathrm{mM}$ phosphate buffer $\mathrm{pH} 7.0$ (GPX) or $0.1 \mathrm{M}$ phosphate buffer $\mathrm{pH} 7.8$ with $1 \mathrm{mM}$ ascorbic acid (APX), in each case containing protease inhibitor cocktail (Sigma-Aldrich). After centrifugation $10 \mathrm{~min}$ at $10000 \times g$ at $4^{\circ} \mathrm{C}$ soluble proteins were desalted using Amicon Ultra Centrifugal Filters (Merck Millipore). Protein concentration was estimated using Roti $^{\circledR}$-Nanoquant Protein quantitation assay (Carl Roth).

Catalase activity was measured according to the method described by Aebi (1984). The decomposition of $10 \mathrm{mM} \mathrm{H}_{2} \mathrm{O}_{2}$ in phosphate buffer (50 $\mathrm{mM}$ phosphate buffer $\mathrm{pH} 7.0$ ) was monitored for $2 \mathrm{~min}$ at $240 \mathrm{~nm}$. Calculations used an absorbance coefficient of $43 \mathrm{M}^{-1} \mathrm{~cm}^{-1}$.

Ascorbate peroxidase activity was determined according to Nakano and Asada (1981). The decrease in absorbance at $290 \mathrm{~nm}$ due to ascorbate oxidation was recorded. The assay mixture contained $20 \mu \mathrm{g}$ proteins, $0.1 \mathrm{mM}$ EDTA, $0.5 \mathrm{mM}$ ascorbic acid, $50 \mathrm{mM}$ and $0.1 \mathrm{mM} \mathrm{H}_{2} \mathrm{O}_{2}$ in phosphate buffer ( $\mathrm{pH}$ 7.0). The absorbance was recorded after $3 \mathrm{~min}$. The APX activity was calculated using the absorbance coefficient of $2.8 \mathrm{mM}^{-1} \mathrm{~cm}^{-1}$.

Glutathione peroxidase activity was determined as the decrease in absorbance at $340 \mathrm{~nm}$ due to the oxidation of NADPH $\left(\varepsilon=6.22 \mathrm{mM}^{-1} \mathrm{~cm}^{-1}\right.$ ) according to Hopkins and Tudhope (1973). The reaction mixture consisted of $50 \mathrm{mM}$ phosphate buffer ( $\mathrm{pH}$ 8.0) containing $0.5 \mathrm{mM}$ EDTA, $0.125 \mathrm{mM}$ NADPH, $1 \mathrm{mM}$ reduced glutathione, $0.5 \mathrm{U} / \mathrm{mL}$ glutathione reductase (Sigma-Aldrich), $300 \mu \mathrm{M}$ tert-butyl hydroperoxide and $20 \mu \mathrm{g}$ proteins. The reaction was monitored for $3 \mathrm{~min}$.

\section{Glucosinolate Content}

The total glucosinolates were extracted and assessed basically as described by Aghajanzadeh et al. (2014). Leaf samples (0.1 g) were boiled for $2 \mathrm{~min}$ in $3 \mathrm{ml} \mathrm{90 \%} \mathrm{methanol.} \mathrm{The} \mathrm{extract} \mathrm{was}$ centrifuged $(2500 \times g$ for $2 \mathrm{~min})$ and the residues were extracted again. The determination of total glucosinolate content was based on color complex formation between glucosinolates and sodium tetrachloropallade (II). The $60 \mu \mathrm{l}$ of extract was incubated for $30 \mathrm{~min}$ in $1800 \mu \mathrm{l} 2 \mathrm{mM} \mathrm{Na}_{2} \mathrm{PdCl}_{4}$ and absorbance of developed color was measured at $450 \mathrm{~nm}$. The glucosinolate content was calculated using standard curve of sinigrin (0-3 mM; SigmaAldrich).

\section{Plant Hormone Determination}

For hormone analysis A. thaliana and E. salsugineum leaf samples were collected at midday. Extraction and analysis were performed according to Dobrev and Kamínek (2002) and Dobrev and Vankova (2012). Briefly, approximately $100 \mathrm{mg}$ fresh samples were homogenized and extracted with methanol/water/formic acid $(15 / 4 / 1, \mathrm{v} / \mathrm{v} / \mathrm{v})$. The following labeled internal standards (10 pmol per sample) were added: ${ }^{2} \mathrm{H}_{6}-\mathrm{ABA},{ }^{2} \mathrm{H}_{3}-\mathrm{PA},{ }^{2} \mathrm{H}_{4}-\mathrm{SA}$, ${ }^{2} \mathrm{H}_{5}$-JA (Olchemim, Czech Republic). Extracts were purified using SPE-C18 column (SepPak-C18, Waters, Milford, MA, USA) and separated on a reverse phase-cation exchange column (Oasis-MCX, Waters). The hormone fraction was eluted with methanol, separated by HPLC (Ultimate 3000, Dionex/Thermo Fisher Scientific, Austria) and the hormones were quantified using a hybrid triple quadrupole/linear ion trap mass spectrometer (3200 Q TRAP, Applied Biosystems/MDS SCIEX, Foster City, CA, USA). The analyses were carried out in three biological replicates. The analysis of $\mathrm{ABA}$ and its catabolites DPA (dihydrophaseic acid) and PA (phaseic acid); ACC (1-aminocyclopropane-1-carboxylic acid), SA (salicylic acid), jasmonic acid (JA) and jasmonate precursor cis-OPDA [cis-(+)-12-oxo-phytodienoic acid] and jasmonoyl-L-isoleucine (JA-Ile) was performed.

\section{Statistical Analysis}

All analysis were calculated with Student's $t$-test. Significant differences are marked at $P \leq 0.05$.

\section{RESULTS}

\section{Production of $\mathrm{H}_{2} \mathrm{O}_{2}$ in Isolated Thylakoids and in Leaves}

In thylakoids from A. thaliana and E. salsugineum an opposite patterns of ROS production were demonstrated by EPR method (Figure 1). Illuminated thylakoids of E. salsugineum generated more $\mathrm{H}_{2} \mathrm{O}_{2}$, as detected indirectly with POBN spin trap which reacts with $\mathrm{H}_{2} \mathrm{O}_{2}$-derived hydroxyl radical (Figures 1A,B), but less $\mathrm{O}_{2}{ }^{-}$, as detected with DMPO spin trap (Figures 1C,D), in comparison to the thylakoids of $A$. thaliana. Addition of $\mathrm{NaN}_{3}$ strongly increased the production of $\mathrm{H}_{2} \mathrm{O}_{2}$, but did not significantly influence generation of $\mathrm{O}_{2}{ }^{-}-$the superoxide anion radical.

To verify unequivocally, whether a difference in $\mathrm{H}_{2} \mathrm{O}_{2}$ generation between the two species is significant also for chloroplasts and leaves we used several methodological approaches. The presence of $\mathrm{H}_{2} \mathrm{O}_{2}$ in the chloroplasts has been tested with a specific $\mathrm{CeCl}_{3}$ staining visualized by TEM. Chloroplasts of mesophyll cells from water-treated A. thaliana revealed no cerium perhydroxide deposits (Figure 2A), while salt stress associated $\mathrm{H}_{2} \mathrm{O}_{2}$ accumulation was visible as black precipitate spots (Figure 2B), mainly at the edges of granal thylakoids (Figure 2C). In contrast, in chloroplasts of $E$. 
A

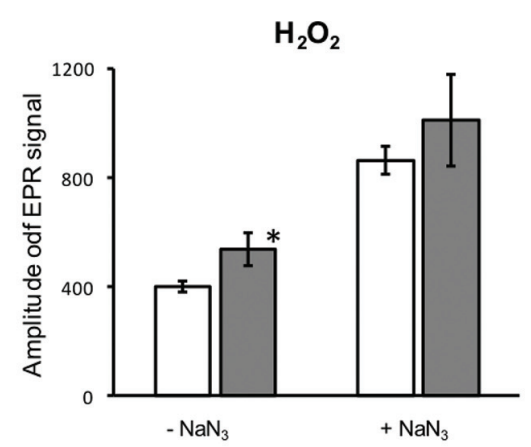

C

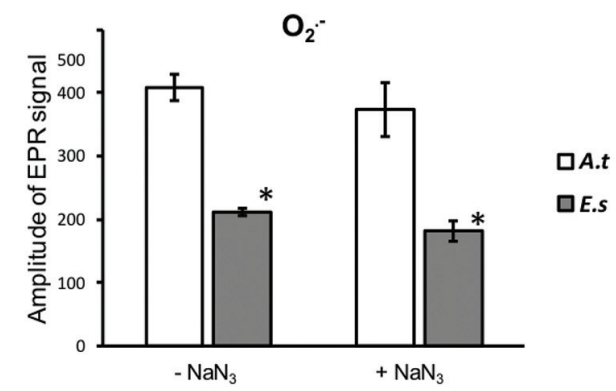

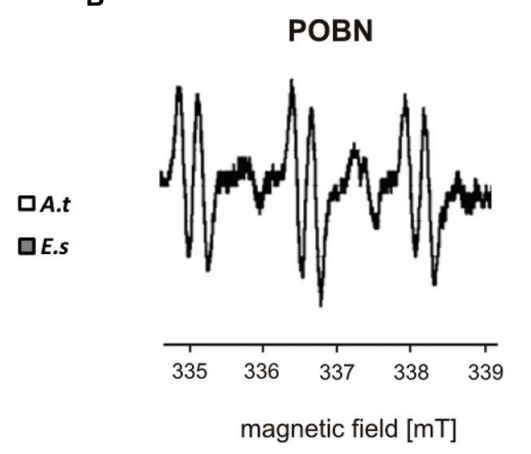

D

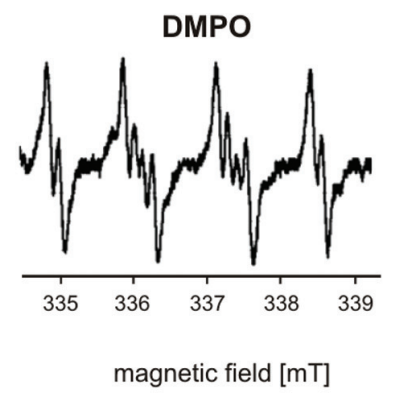

FIGURE 1 | Electron paramagnetic resonance (EPR) spin-trapping spectroscopy measurements of $\mathrm{H}_{2} \mathrm{O}_{2}(A, B)$ and $\mathrm{O}_{2}^{\bullet}-(C, D)$ production by illuminated thylakoids isolated from water-treated Arabidopsis thaliana and Eutrema salsugineum. (A) The intensity of EPR signal of $\mathrm{H}_{2} \mathrm{O}_{2}$-derived hydroxyl radicals; (B) representative EPR spectra of the POBN adduct; (C) The intensity of superoxide anion radical measured with DMPO-OOH spin-trap; (D) representative EPR spectra of the DMPO adduct. The analyses were performed in the presence and absence of sodium azide (NaN ${ }_{3}$ ). 'Asterisk' indicates significant difference from $A$. thaliana. Each data point represents the mean $\pm \mathrm{SD}(n=3)$. Significant differences between the averaged $\mathrm{H}_{2} \mathrm{O}_{2}$ production with and without $\mathrm{NaN}_{3}$ in both species are not marked on the graph.

salsugineum an electron-dense precipitates were detectable already in control (Figure 2D), while more pronounced dark spots were visible after salt stress (Figures 2E,F). Another difference between $A$. thaliana and E. salsugineum detected by TEM was that a salinity-induced thylakoid swelling was detected only in A. thaliana chloroplasts (Figure 2B), while in $E$. salsugineum chloroplasts no signs of such destruction occurred (Figures 2E,F). Staining controls without $\mathrm{CeCl}_{3}$ showed no electron-dense deposits in both species (data not shown).

Similarly, in leaves, enhanced content of $\mathrm{H}_{2} \mathrm{O}_{2}$ was documented in E. salsugineum than in A. thaliana (Figure 3Astaining with Amplex Red, and Figure 3B-staining with DAB). However, in spite of the high availability of this ROS a very low level of MDA was detected in E. salsugineum leaves, indicating a low extent of oxidative damage to membrane lipids (Figure 4A). This difference between the two species disappeared when isolated TMs were compared (Figure 4B). On this basis we formulated a hypothesis that enhanced leakage of $\mathrm{H}_{2} \mathrm{O}_{2}$ from plastids keeps the antioxidant system up-regulated, thereby preadapting plants to the salinity stress.

\section{Comparison of Antioxidant System in Arabidopsis and Eutrema}

The capacity of antioxidant system was compared in leaves of A. thaliana and E. salsugineum in the control conditions and after salinity stress. Due to the disturbed kinetics of enzymatic assays observed in crude leaf extracts (LEs) from E. salsugineum, the activities of $\mathrm{H}_{2} \mathrm{O}_{2}$ scavenging enzymes were analyzed in purified protein fractions. The activities of CAT were similar in both species in the control conditions and remained unchanged after salinity stress (Figure 5A). The activity of APX was lower in the E. salsugineum both in control and after salinity stress (Figure 5B). In contrast, the activity of thiol-dependent GPX was higher in E. salsugineum both in control and after a salinity stress (Figure 5C).

Considering the chloroplastic generation of $\mathrm{H}_{2} \mathrm{O}_{2}$ in $E$. salsugineum, the antioxidant system in this organelle might be of particular importance for this species. Therefore, we compared the antioxidant enzymes present at TMs and in the soluble fraction (LEs; Figure 6). The stromal form of APX (sAPX) was present in similar amount in both species, while distribution of thylakoid bound APX (tAPX) showed a clear difference between A. thaliana and E. salsugineum. This form was much more abundant in Eutrema both in controls and in salinity-treated plants, while it was barely detected in A. thaliana. Another differences between these two species was related to FeSOD. In E. salsugineum, FeSOD was less abundant in LEs, while it was much more abundant in TMs, when compared with $A$. thaliana (Figure 6). To get an insight into the level of thiols and thiol-dependent enzymes we compared the 


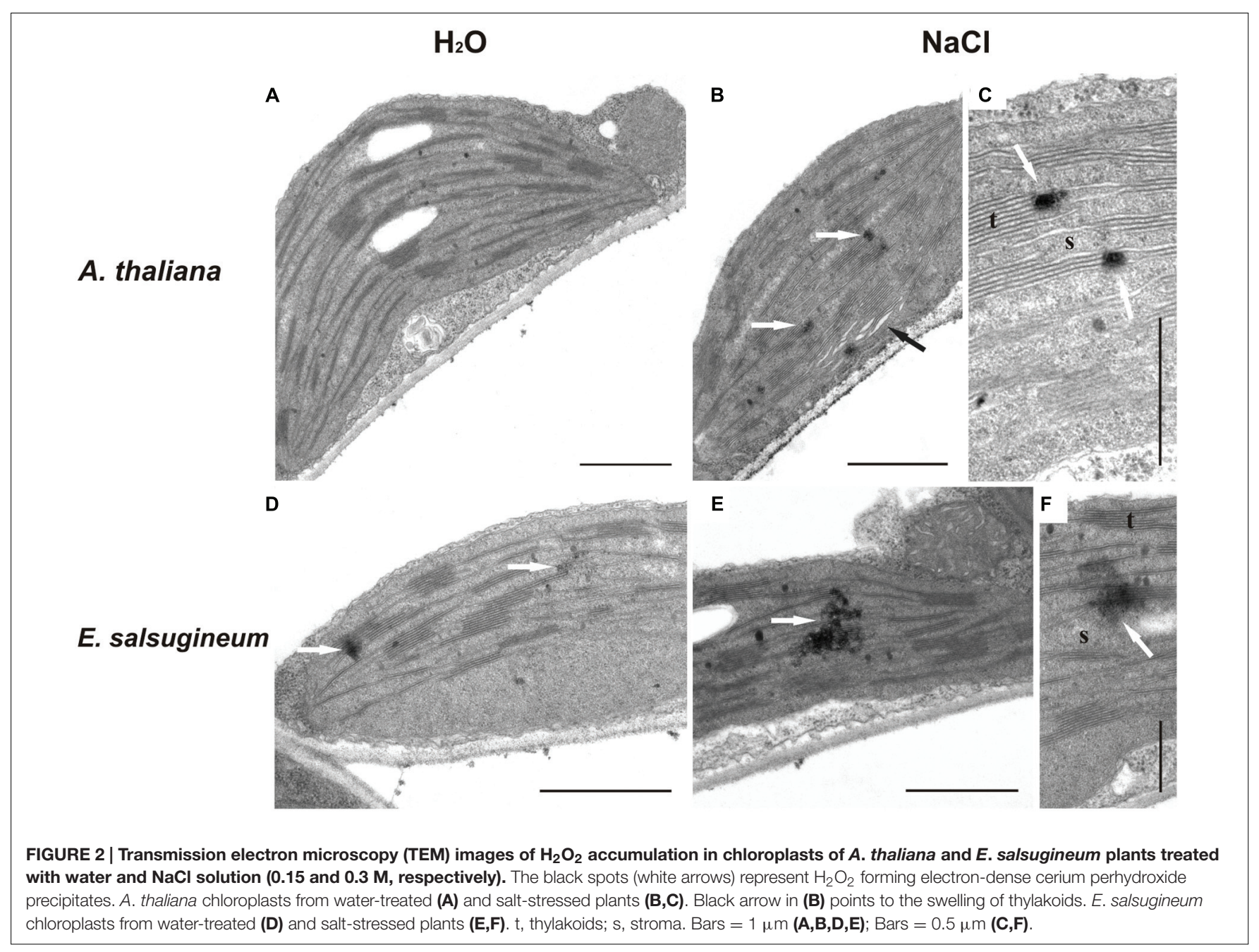

amount of stromal peroxiredoxins Q (PrxQ) and the enzyme of reduced glutathione (GSH) synthesis, gamma glutamylcysteine synthase ( $\gamma$-ECS). In E. salsugineum PrxQ were more abundant than in A. thaliana, and a slight decrease was noted after salinity treatment in both species, whilst $\gamma$-ECS was present at similar amount in both species (Figure 6). These pronounced differences between E. salsugineum and A. thaliana in the amounts of various ROS scavengers in chloroplasts suggest that there is a stronger need to protect photosynthetic membranes against ROS in the former species, already in the absence of stress.

\section{Signaling Pathways Associated with ROS}

It is well-documented that ROS signaling is interconnected with stress hormones (Baxter et al., 2014). Therefore, we analyzed the endogenous levels of stress-related hormones in A. thaliana and E. salsugineum plants grown in control conditions and changes evoked by salt-treatment. In control conditions, the concentrations of $\mathrm{ABA}$ and its catabolite PA were similar in leaves of both species (Figures 7A,B), while the level of another ABA catabolite, DPA was twofold lower in E. salsugineum (Figure 7C). Salt treatment caused an increase in ABA (twofold),
DPA (twofold) and PA (threefold) in A. thaliana, whereas no significant changes (ABA, DPA), and a slight increase in (PA), were detected in E. salsugineum (Figures 7A-C). Concentrations of ethylene precursor ACC were similar in the two species in control conditions and underwent a similar increase due to salinity (Figure 7D). Concentrations of SA and jasmonates were much lower in the control E. salsugineum in comparison to A. thaliana: SA almost fourfold, JA by 4.5 -fold, Ja-Ile by $2.4-$ fold and cisOPDA by 5.5 -fold (Figures 7E-H). NaCl-treatment increased the levels of these hormones in A. thaliana (1.7-, 2.6, 6.6-, 1.8-fold, respectively) but not in E. salsugineum, where their decreased concentrations were found (1.8-, 4-, 4-, 3.2-fold, respectively).

One of the highlights of $\mathrm{H}_{2} \mathrm{O}_{2}$ signaling from chloroplasts seems to be enhanced synthesis of glucosinolates, as demonstrated in GO5 plants (Balazadeh et al., 2012; Sewelam et al., 2014). To check this cue in our wild type model, we measured the total concentration of leaf glucosinolates. The total level of glucosinolates was enhanced in leaves of E. salsugineum in comparison with $A$. thaliana in control conditions, and a stronger increase was detected in E. salsugineum after salinity treatment (Figure 8). 


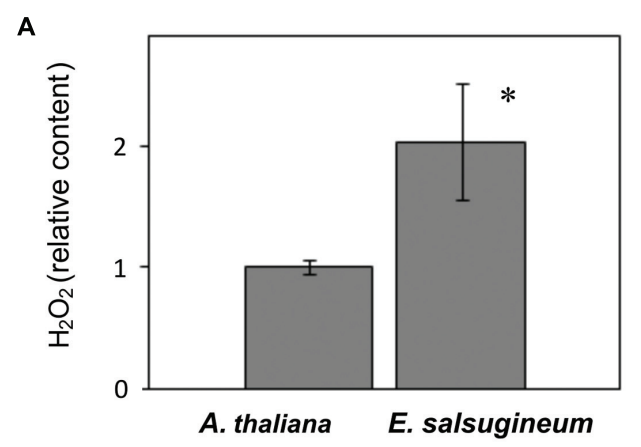

B

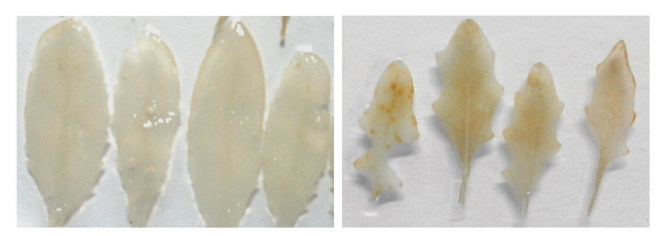

A. thaliana

E. salsugineum

FIGURE 3 | Hydrogen peroxide $\left(\mathrm{H}_{2} \mathrm{O}_{2}\right)$ accumulation in leaves from water-treated $A$. thaliana and $E$. salsugineum plants. (A) Content of $\mathrm{H}_{2} \mathrm{O}_{2}$ in deproteinated leaf extracts determined using the Amplex Red assay. Results were normalized to the average $\mathrm{H}_{2} \mathrm{O}_{2}$ content in $A$. thaliana. Data represent the mean $\pm \mathrm{SD}(n=3)$. 'Asterisk' indicates significant difference. (B) Visualization of $\mathrm{H}_{2} \mathrm{O}_{2}$ accumulation assessed by DAB staining of representative leaves.
A

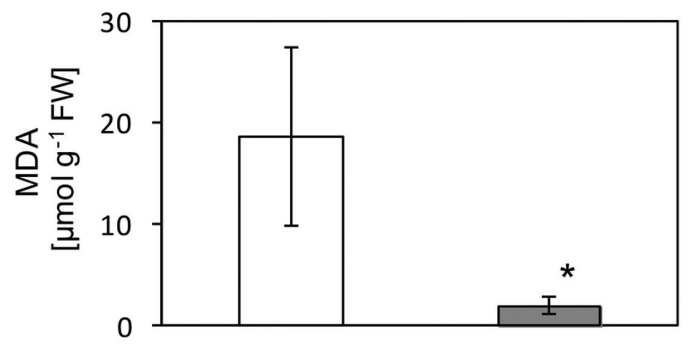

B

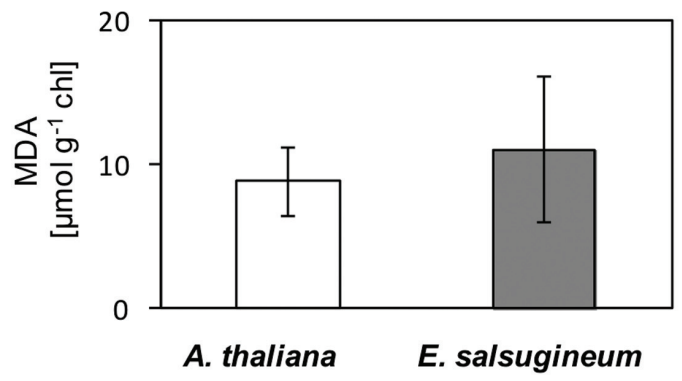

FIGURE 4 | Content of malondialdehyde (MDA) in leaves of water-treated $A$. thaliana and $E$. salsugineum plants $(A)$ and isolated thylakoid membranes (TMs; B) assessed by high-performance liquid chromatography (HPLC). Each data point represents the mean $\pm \mathrm{SD}$ $(n=3)$. 'Asterisk' indicates significant difference from $A$. thaliana.

\section{DISCUSSION}

In this work, we compared a halophytic E. salsugineum with glycophytic $A$. thaliana in regard to the chloroplastic $\mathrm{H}_{2} \mathrm{O}_{2}$ signature on the salt stress tolerance. As a first step, we verified whether a chloroplastic $\mathrm{H}_{2} \mathrm{O}_{2}$ signal, previously reported with use of isolated TMs (Wiciarz et al., 2015), persists in the relatively undamaged organelle and tissue.

Enhanced generation of $\mathrm{H}_{2} \mathrm{O}_{2}$ within the chloroplasts of control and stress-treated Eutrema was visualized by TEM, suggesting that a higher level of $\mathrm{H}_{2} \mathrm{O}_{2}$ persists in the whole chloroplasts. However, it is not uniformly distributed but spotted in the proximity of granal thylakoids, were it is produced. TEM images also revealed, that $\mathrm{NaCl}$ treatment caused thylakoid swelling only in A. thaliana. Thylakoid dilation is a welldocumented symptom of salinity stress in plants (Niewiadomska et al., 2011; Yamane et al., 2012; Pottosin and Shabala, 2016), while structural integrity of E. salsugineum chloroplasts under mild NaCl-stress was previously shown by Chang et al. (2015). This lack of swelling might be attributed to the activity of thylakoid ion channels, and/or $\mathrm{K}^{+}\left(\mathrm{Na}^{+}\right) / \mathrm{H}^{+}$antiporters which control the ion homeostasis under salinity-stress (Pottosin and Shabala, 2016).

Although we did not find any direct evidence for an export of $\mathrm{H}_{2} \mathrm{O}_{2}$ from plastids, the total $\mathrm{H}_{2} \mathrm{O}_{2}$ level in leaf extracts was also elevated in Eutrema leaves. Hence, it is likely that a chloroplast
$\mathrm{H}_{2} \mathrm{O}_{2}$ generation contributes considerably to this phenomenon. Our speculation is based on a well-accepted phenomenon that illuminated chloroplast is a major site of ROS production in the mesophyll cells (Asada, 2006). Moreover, a considerable leakage of $\mathrm{H}_{2} \mathrm{O}_{2}$ from healthy chloroplasts has been shown experimentally (Mubarakshina et al., 2010; Borisova et al., 2012).

Here, performed study revealed that an increased generation of $\mathrm{H}_{2} \mathrm{O}_{2}$ in halophytic species is associated by a decreased generation of $\mathrm{O}_{2}^{\bullet-}$. Considering a much higher reactivity of $\mathrm{O}_{2}^{\bullet}-$ than $\mathrm{H}_{2} \mathrm{O}_{2}$, this pattern of ROS production might shape a specific ROS signature important for so called stressprepardeness of Eutrema. This speculation is supported by a much lower oxidative damage (MDA) in leaves of E. salsugineum than in A. thaliana. As argued by Tiwari et al. (2013), superoxide, but not $\mathrm{H}_{2} \mathrm{O}_{2}$, is required to evoke oxidative damage in photosynthetic membranes. This damage is mediated by the hydroxyl radical and is manifested by the appearance of carboncentered radical. Experiments with the flu A. thaliana mutant and the $f l u$ overexpressing tAPX, enabled to demonstrate that $\mathrm{H}_{2} \mathrm{O}_{2}$ antagonizes the ${ }^{1} \mathrm{O}_{2}$-mediated cell death response and growth inhibition, which might be particularly advantageous under stress (Laloi et al., 2007). A rapid conversion of a more harmful ROS (such as $\mathrm{O}_{2}^{\bullet-}$ ) into a longer-living and less toxic $\mathrm{H}_{2} \mathrm{O}_{2}$ has previously been concluded for halophytic plants on the basis of enhanced activity of superoxide dismutase (Bose et al., 2014). In halophytic E. salsugineum an operation 
A

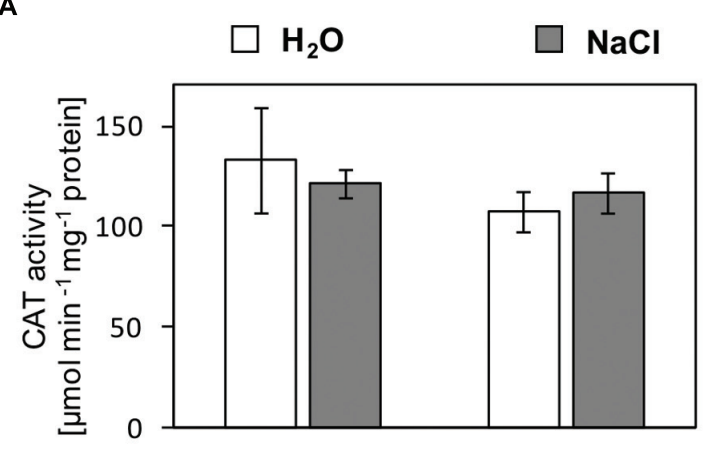

B

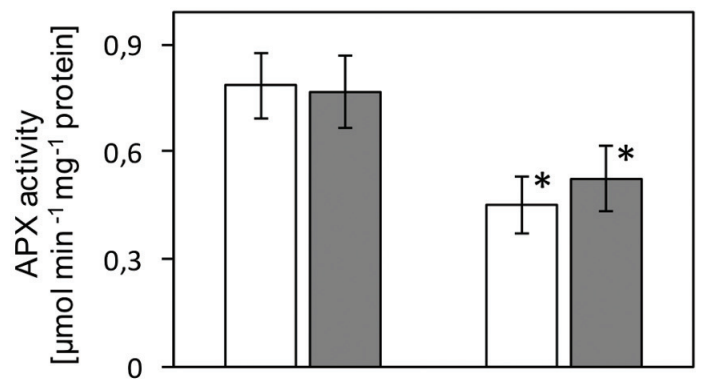

C

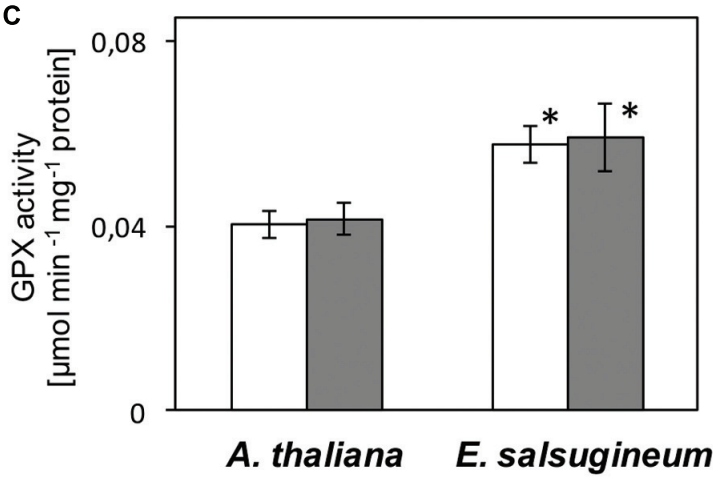

FIGURE 5 | Activities of CAT (A), APX (B), GPX (C) enzymes in the whole leaf cells of $A$. thaliana and $E$. salsugineum plants treated with water and $\mathrm{NaCl}$ solution ( 0.15 and $0.3 \mathrm{M}$, respectively). Data represent mean $\pm \mathrm{SD}(n=3)$. 'Asterisk' indicates significant difference from $A$. thaliana from the same treatment.

of the protective electron cycling around PSII (Stepien and Johnson, 2009; Wiciarz et al., 2015) seems to support that view. Such a cycle could be beneficial for plants in several ways. Firstly, it may create a more safe electron sink in comparison to the production of superoxide and its toxic derivatives. Secondly, an abundance of $\mathrm{H}_{2} \mathrm{O}_{2}$ may play a signaling role.

A growing body of data indicate that $\mathrm{H}_{2} \mathrm{O}_{2}$ triggers defense responses in plant cells (for a recent review, see Suzuki et al., 2012; Baxter et al., 2014; Bose et al., 2014; Ismail et al., 2014). In this respect, ROS formation in chloroplast may also act indirectly via strengthening or amplifying retrograde redox signals (Galvez-Valdivieso and Mullineaux, 2010). One of the

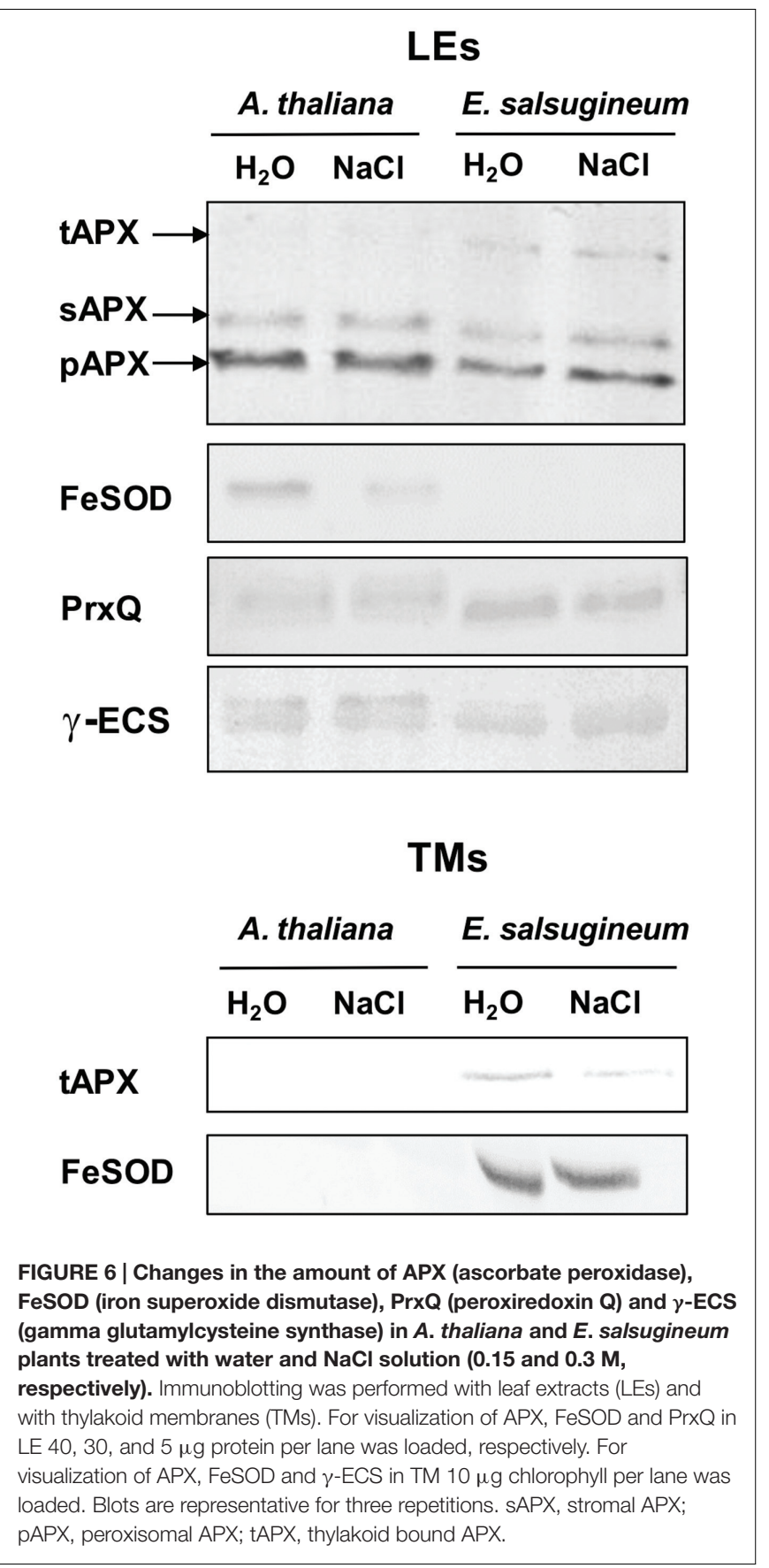

targets of ROS signaling route under stress is an up-regulation of antioxidants (Fryer et al., 2003; Geisler et al., 2006). However, the activity of a major $\mathrm{H}_{2} \mathrm{O}_{2}$ scavenger CAT was similar in E. salsugineum and A. thaliana in controls and in salinitytreated plants, whereas APX activity was even decreased in E. salsugineum. This is in agreement with numerous reports on salt cress. The transcript profiling analysis with two $E$. salsugineum ecotypes did not detect any antioxidant genes among those specifically up-regulated by salinity (Taji et al., 2004; Wong et al., 2006). Also, a proteomic data did not detect any salinity-dependent stimulation of antioxidant enzymes in 


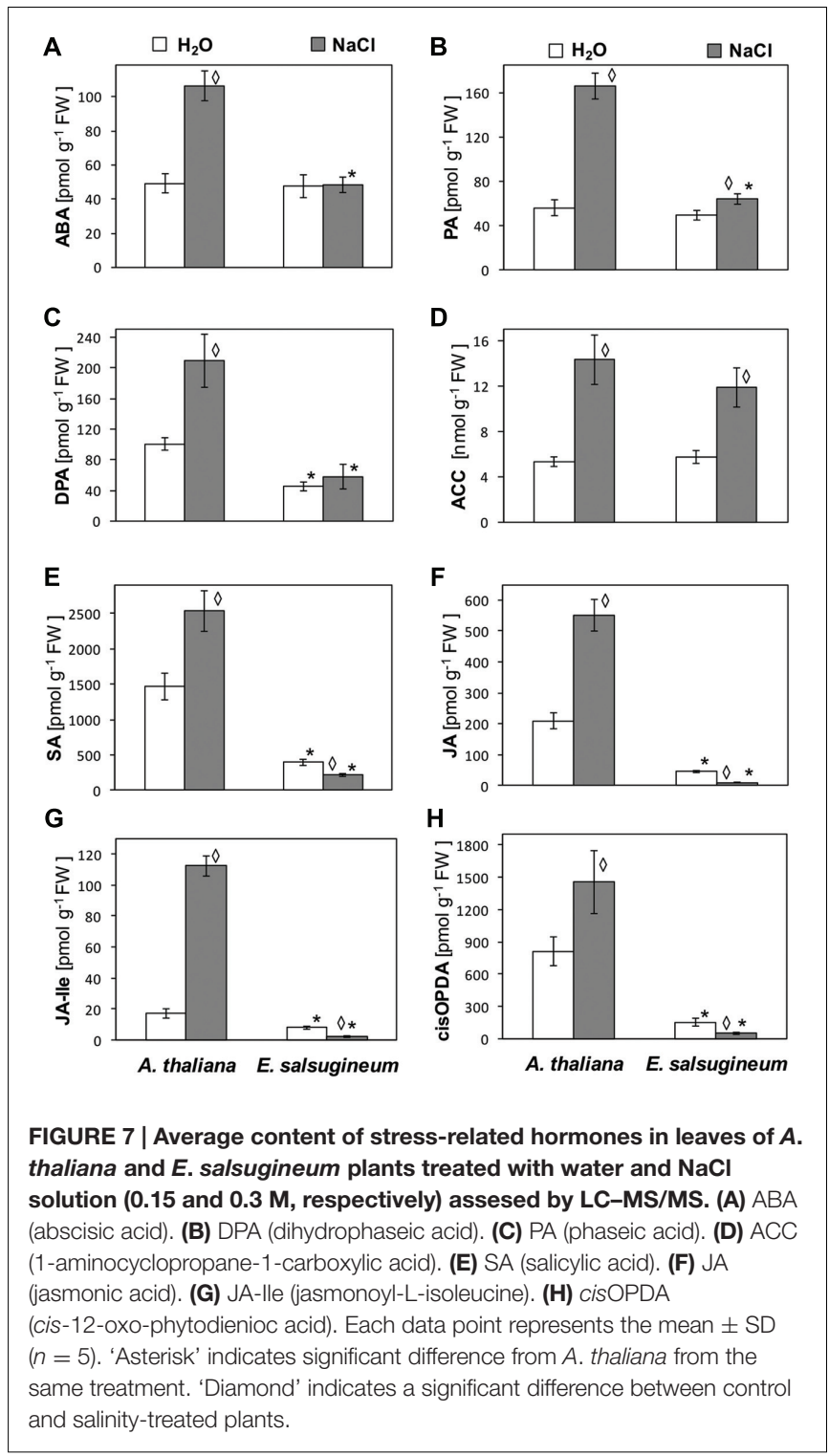

this species (Pang et al., 2010). At activity level M'rah et al. (2006) previously noted that stimulation of CAT activity in $E$. salsugineum occurred only under a mild salinity treatment, not greater than $100 \mathrm{mM}$, whereas some stimulation was related to total peroxidase activity (assessed with guaiacol as electron donor).

Intriguingly, all these data suggest that a high stress resistance of Eutrema does not rely on the major enzymatic $\mathrm{H}_{2} \mathrm{O}_{2}$ scavengers of mesophyll cells. We argue, that data obtained with a partially purified (desalted) protein extracts (as used here) give a more precise information about changes in the enzymatic activities in comparison to the data typically obtained with crude extracts. The latter are strongly influenced by a different availability of low molecular compounds, such as ascorbate in case of APX, and glutathione in case of GPX. Indeed, an increased pool of reduced ascorbate and of total glutathione was found due to salinity in Eutrema, but not in Arabidopsis (Wiciarz

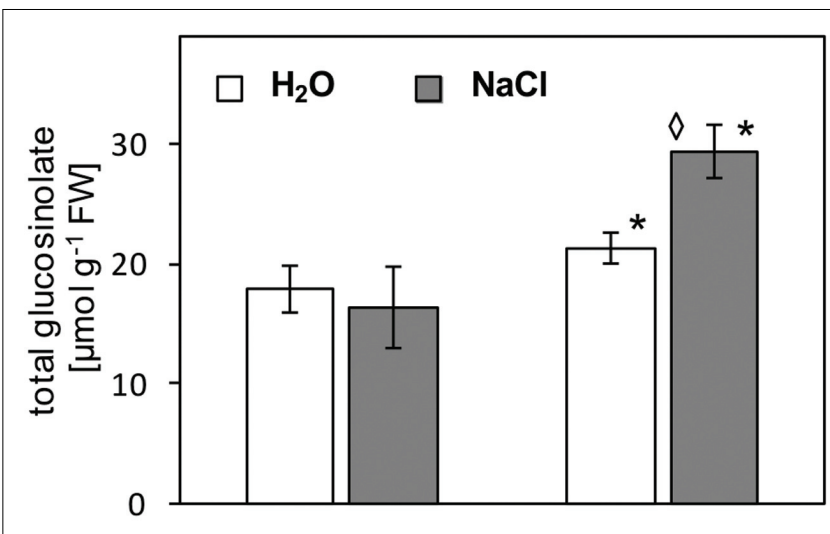

A. thaliana E. salsugineum

FIGURE 8 | Total glucosinolates content in leaves of $A$. thaliana and $E$. salsugineum plants treated with water and $\mathrm{NaCl}$ solution $(0.15$ and $\mathbf{0 . 3} \mathbf{M}$, respectively). Each data point represents the mean $\pm \mathrm{SD}(n=3)$. 'Asterisk' indicates significant difference from $A$. thaliana from the same treatment. 'Diamond' indicates significant difference between control and salinity-treated plants.

et al., 2016). This feature might be important in the context of $\mathrm{H}_{2} \mathrm{O}_{2}$ signaling. As suggested by Bose et al. (2014), decreased activities of a major $\mathrm{H}_{2} \mathrm{O}_{2}$ scavengers allow for a persistence of ROS signal in the cells. A fine-tuning of specific antioxidants seems to be more important for stress tolerance than a bulk changes in the antioxidant status (Jiang et al., 2007; Pang et al., 2010). We are, however, aware that a relation between ROS production and scavenging is highly dynamic while a situation reported here for Eutrema relates only to certain time point. But, on the other hand, it was verified with use of several methods and plants sets to be considered as physiologically relevant.

Among the enzymatic $\mathrm{H}_{2} \mathrm{O}_{2}$ scavengers only GPX was enhanced in E. salsugineum. This points to the importance of redox active thiols. GPXs reduce not only $\mathrm{H}_{2} \mathrm{O}_{2}$ but also organic hydroperoxides using reducing power of GSH. Hence, they may contribute to the dramatically reduced MDA level. A noteworthy stimulation of antioxidant defense associated with glutathione has previously been revealed in E. salsugineum by transcript profiling of Gong et al. (2005). This was represented by GPXs (GPX2 and GPX6), glutaredoxins and glutathione-S-transferases (GSTZ1 and GSTU7). Four out of eight GPXs found in E. salsugineum have been shown to respond to salt and osmotic stresses at both transcript and protein level (Gao et al., 2014). It is worthy to note here, that GPX may serve as a specific sensor and transducer of $\mathrm{H}_{2} \mathrm{O}_{2}$ signal. A dual function of GPX associated with both scavenging of ROS and transferring the $\mathrm{H}_{2} \mathrm{O}_{2}$ signal has been pointed out repeatedly (Delaunay et al., 2002; Miao et al., 2006). Moreover, a specific activation of GPX1 promoter in tobacco by a chloroplast-generated $\mathrm{H}_{2} \mathrm{O}_{2}$ was documented by Avsian-Kretchmer et al. (2004). Whereas, study with Arabidopsis $g p \times 3$ mutant demonstrated that GPX3 participates in the ABA and $\mathrm{H}_{2} \mathrm{O}_{2}$ signaling pathway regulating the stomatal aperture and resistance to drought (Miao et al., 2006). 
Considering an enhanced generation of $\mathrm{H}_{2} \mathrm{O}_{2}$ and changed $\mathrm{O}_{2}{ }^{\bullet}-/ \mathrm{H}_{2} \mathrm{O}_{2}$ ratio in chloroplasts, a major role might be attributed to the anti-oxidative protection within this organelle. Indeed, TMs of Eutrema appeared to be better protected against ROS in comparison with Arabidopsis, as indicated by a higher abundance of tAPX and FeSOD. This corresponds with enhanced level of FeSOD transcripts in the control E. salsugineum, reported by Taji et al. (2004). In regard to chloroplast stroma, we found an enhanced amount of PrxQ in E. salsugineum. These above-mentioned features of chloroplast antioxidant system are constitutive in E. salsugineum, which supports earlier conclusions on the stress preparedness of this species (Inan et al., 2004; Taji et al., 2004; Gong et al., 2005; Wong et al., 2006). They are also in line with the view that components of redox homeostasis in chloroplasts are crucial for salinity tolerance (Niewiadomska and Wiciarz, 2015; Uzilday et al., 2015). So far, recognized chloroplast redox players highly engaged in E. salsugineum are: adenosine $5^{\prime}$-phosphosulfate reductase involved in sulfate assimilation (Gong et al., 2005); thioredoxin CDSP32 (M'rah et al., 2007); 2-cys peroxiredoxin BAS1 (Gao et al., 2009) and plastid terminal oxidase (PTOX), an enzyme of chlororespiratory pathway (Stepien and Johnson, 2009; Wiciarz et al., 2015). In addition to these, also proteins associated with the Calvin-Benson cycle, such as a more active Rubisco (Wiciarz et al., 2015) and a more abundant glyceraldehyde 3-phosphate dehydrogenase (Pang et al., 2010; Chang et al., 2015) are likely to contribute to the improved redox balance in $E$. salsugineum by efficient recycling of $\mathrm{ADP}$ and $\mathrm{NADP}^{+}$.

A very weak response to salinity stress at the level of tested antioxidants may raise a question: whether E. salsugineum (and A. thaliana) senses the salinity stress at all? This has been clarified by analysis of stress hormones. A massive increase in stress-related hormones (ABA, SA, JA, and ethylene precursor) was detected in salinity-treated A. thaliana. These hormones are responsible for several stress effects in plants, among them stomatal closure and retardation of growth, although, their action is quite complex and may lead either to survival or to cell death. In contrast, in Eutrema the only indicator of salinity-evoked stress was a stimulation of ethylene synthesis. Although, some enhancement in ABA catabolite PA, may suggest a previous ABA induction. Earlier study of Ellouzi et al. (2014) documented that levels of ABA, JA, and ACC were increased in E. salsugineum after 2 days of salt stress, while $400 \mathrm{mM} \mathrm{NaCl}$ treatment for more than 5 days caused minimal changes in $\mathrm{ABA}$ and JA concentrations (Taji et al., 2004; Arbona et al., 2010). In E. salsugineum the levels of lipid-based active jasmonates (JA and JA-Ile) were low and even decreased due to salinity-treatment. This effect seems to be closely related to salt-resistance of Eutrema. As shown by Ismail et al. (2014), an interplay between ABA and JA is of particular importance for plant's survival or death under salinity stress. A comparison of the two Vitis species differing in salt-stress tolerance revealed that in the more salt-tolerant Vitis rupestris ABA accumulated early and strongly suppressed the formation of JA-Ile, while in the salt-sensitive $V$. vinifera the accumulation of $\mathrm{ABA}$ was delayed and correlated which allow for accumulation of JA-Ile up to the high levels. A suppression of jasmonates in E. salsugineum might also be explained in accordance with the data obtained with AtGO5 mutant overproducing $\mathrm{H}_{2} \mathrm{O}_{2}$ in chloroplasts, in which a stimulated expression of several repressors of JA signaling pathway has occurred (Balazadeh et al., 2012). A very weak response to salinity at the level of ABA in E. salsugineum in contrast to $A$. thaliana is surprising in view of data on a higher expression of genes for ABA-biosynthesis and signaling (Taji et al., 2004; Gong et al., 2005; Wu et al., 2012) and an increased number of genes involved in ABA synthesis pathway in Eutrema genome (Wu et al., 2012). Possibly, in E. salsugineum the increased level of ABA is confined to the early stage of development or this hormone is very rapidly modulated in response to stress (indicated by slight increase in PA). A weak response of stress hormones to salinity in E. salsugineum supports the earlier conclusion of Hou and Bartels (2015), that comparing with $A$. thaliana the higher $\mathrm{NaCl}$ levels $(600 \mathrm{mM})$ are required in E. salsugineum to trigger some defense reactions, such as increased transcription of two aldehyde dehydrogenase genes.

On the other hand, the importance of glucosinolates for stress-resistance of E. salsugineum is depicted by their enhanced level and a strong salinity-dependent increase. A great variation in the patterns of aliphatic, aromatic, and indole glucosinolate was documented in E. salsugineum, depending on the organ and developmental stage (Pang et al., 2012). However, a precise role of these compounds in stress tolerance is not clarified yet. An up-regulation of the synthesis of indole glucosinolates and phytoalexin camalexin was found as a specific feature of increased $\mathrm{H}_{2} \mathrm{O}_{2}$ signaling from chloroplasts in studies with AtGO5 mutant made by Balazadeh et al. (2012) and Sewelam et al. (2014). However, E. salsugineum does not produce camalexin, but wasalexins $\mathrm{A}$ and $\mathrm{B}$ and methoxybrassenin B (Pedras and Adio, 2008). Intriguingly, as another support for a constitutive stress-preparedness of Eutrema, the three phytoalexins (indolyl-3-acetonitrile, caulilexin C, and arvelexin) are found as phytoanticipins, i.e., they are constitutively produced in this species (Pedras and Adio, 2008).

\section{CONCLUSION}

In this paper we provided evidence for a specific pattern of ROS formation and accumulation in halophytic Eutrema in comparison to glycophytic Arabidopsis. In concert with that, a pronounced changes in the antioxidant system were revealed in E. salsugineum, such as a decreased activity of APX, an increased activity of GPX, an increased level of PrxQ, and the presence of thylakoid-bound forms of FeSOD and APX. All these cues allow for enhanced $\mathrm{H}_{2} \mathrm{O}_{2}$ signaling from chloroplasts of E. salsugineum already in the control conditions, at decreased level of oxidative damage in the same time. This signaling led to enhanced level of glucosinolates in Eutrema and decreased levels of stress hormones (SA and jasmonates). Salinity-stress evoked a strong up-regulation of all tested stress hormones in A. thaliana whereas in E. salsugineum only a stimulation in ethylene synthesis and $A B A$ catabolism was noted. On this basis we hypothesize that $\mathrm{H}_{2} \mathrm{O}_{2}$ signaling is engaged in the halophytic species to modulate a hormonal responses in such a way to minimize the triggering of jasmonate pathway which leads to cell death. 


\section{AUTHOR CONTRIBUTIONS}

MP performed analysis of antioxidants, staining and quantitation of $\mathrm{H}_{2} \mathrm{O}_{2}$, determination of glucosinolates, participated in TEM analysis and in preparation of the manuscript. MW measured MDA, participates in the analysis of antioxidants and ROS. IJ measured ROS with EPR. MK-K performed TEM analysis. $\mathrm{PD}$ and RV measured stress hormones, and RV participated in

\section{REFERENCES}

Aebi, H. (1984). Catalase in vitro. Methods Enzymol. 105, 121-126. doi: 10.1016/S0076-6879(84)05016-3

Aghajanzadeh, T., Hawkesford, M. J., and De Kok, L. J. (2014). The significance of glucosinolates for sulfur storage in Brassicaceae seedlings. Front. Plant Sci. 5:704. doi: 10.3389/fpls.2014.00704

Amtmann, A. (2009). Learning from evolution: Thellungiella generates new knowledge on essential and critical components of abiotic stress tolerance in plants. Mol. Plant 2, 3-12. doi: 10.1093/mp/ssn094

Arbona, V., Argamasilla, R., and Gómez-Cadenas, A. (2010). Common and divergent physiological, hormonal and metabolic responses of Arabidopsis thaliana and Thellungiella halophila to water and salt stress. J. Plant Physiol. 167, 1342-1350. doi: 10.1016/j.jplph.2010.05.012

Asada, K. (2006). Production and scavenging of reactive oxygen species in chloroplasts and their functions. Plant Physiol. 141, 391-396. doi: 10.1104/pp.106.082040

Avsian-Kretchmer, O., Gueta-Dahan, Y., Lev-Yadun, S., Gollop, R., and BenHayyim, G. (2004). The salt-stress signal transduction pathway that activates the gpx1 promoter is mediated by intracellular $\mathrm{H} 2 \mathrm{O} 2$, different from the pathway induced by extracellular H2O2. Plant Physiol. 135, 1685-1696. doi: 10.1104/pp.104.041921

Balazadeh, S., Jaspert, N., Arif, M., Mueller-Roeber, B., and Maurino, V. G. (2012). Expression of ROS-responsive genes and transcription factors after metabolic formation of $\mathrm{H} 2 \mathrm{O} 2$ in chloroplasts. Front. Plant Sci. 3:234. doi: 10.3389/fpls.2012.00234

Baxter, A., Mittler, R., and Suzuki, N. (2014). ROS as key players in plant stress signalling. J. Exp. Bot. 65, 1229-1240. doi: 10.1093/jxb/ert375

Bestwick, C. S., Brown, I. R., Bennett, M. H., and Mansfield, J. W. (1997). Localization of hydrogen peroxide accumulation during the hypersensitive reaction of lettuce cells to Pseudomonas syringae pv phaseolicola. Plant Cell 9, 209-221. doi: 10.1105/tpc.9.2.209

Borisova, M. M. M., Kozuleva, M. A., Rudenko, N. N., Naydov, I. A., Klenina, I. B., and Ivanov, B. N. (2012). Photosynthetic electron flow to oxygen and diffusion of hydrogen peroxide through the chloroplast envelope via aquaporins. Biochim. Biophys. Acta 1817, 1314-1321. doi: 10.1016/j.bbabio.2012. 02.036

Bose, J., Rodrigo-Moreno, A., and Shabala, S. (2014). ROS homeostasis in halophytes in the context of salinity stress tolerance. J. Exp. Bot. 65, 1241-1257. doi: $10.1093 /$ jxb/ert430

Chang, L., Guo, A., Jin, X., Yang, Q., Wang, D., Sun, Y., et al. (2015). The beta subunit of glyceraldehyde 3-phosphate dehydrogenase is an important factor for maintaining photosynthesis and plant development under salt stressbased on an integrative analysis of the structural, physiological and proteomic changes in chloroplasts in Thellungiella halophila. Plant Sci. 236, 223-238. doi: 10.1016/j.plantsci.2015.04.010

Delaunay, A., Pflieger, D., Barrault, M. B., Vinh, J., and Toledano, M. B. (2002). A thiol peroxidase is an $\mathrm{H} 2 \mathrm{O} 2$ receptor and redox-transducer in gene activation. Cell 111, 471-481. doi: 10.1016/S0092-8674(02)01048-6

Dobrev, P. I., and Kamínek, M. (2002). Fast and efficient separation of cytokinins from auxin and abscisic acid and their purification using mixed-mode solid-phase extraction. J. Chromatogr. A 950, 21-29. doi: 10.1016/S00219673(02)00024-9

Dobrev, P. I., and Vankova, R. (2012). Quantification of abscisic acid, cytokinin, and auxin content in salt-stressed plant tissues. Methods Mol. Biol. 913, 251261. doi: 10.1007/978-1-61779-986-0_17 writing of the text. EN designed the experiment and wrote the manuscript. All authors have read and approved the manuscript.

\section{FUNDING}

This work was supported by the Polish National Science Centre project (2011/03/B/NZ9/01619 EN, MP, MW).

Ellouzi, H., Hamed, K. B., Hernández, I., Cela, J., Müller, M., Magné, C., et al. (2014). A comparative study of the early osmotic, ionic, redox and hormonal signaling response in leaves and roots of two halophytes and a glycophyte to salinity. Planta 240, 1299-1317. doi: 10.1007/s00425-014-2154-7

Fahnenstich, H., Scarpeci, T. E., Valle, E. M., Flügge, U. I., and Maurino, V. G. (2008). Generation of hydrogen peroxide in chloroplasts of Arabidopsis overexpressing glycolate oxidase as an inducible system to study oxidative stress. Plant Physiol. 148, 719-729. doi: 10.1104/pp.108.126789

Fryer, M. J., Ball, L., Oxborough, K., Karpinski, S., Mullineaux, P. M., and Baker, N. R. (2003). Control of Ascorbate Peroxidase 2 expression by hydrogen peroxide and leaf water status during excess light stress reveals a functional organisation of Arabidopsis leaves. Plant J. 33, 691-705. doi: 10.1046/j.1365313X.2003.01656.x

Gadjev, I., Vanderauwera, S., Gechev, T. S., Laloi, C., Minkov, I. N., Shulaev, V., et al. (2006). Transcriptomic footprints disclose specificity of reactive oxygen species signaling in Arabidopsis. Plant Physiol. 141, 436-445. doi: 10.1104/pp.106.078717

Galvez-Valdivieso, G., and Mullineaux, P. M. (2010). The role of reactive oxygen species in signalling from chloroplasts to the nucleus. Physiol. Plant. 138, 430-439. doi: 10.1111/j.1399-3054.2009.01331.x

Gao, F., Chen, J., Ma, T., Li, H., Wang, N., Li, Z., et al. (2014). The glutathione peroxidase gene family in Thellungiella salsuginea: genome-wide identification, classification, and gene and protein expression analysis under stress conditions. Int. J. Mol. Sci. 15, 3319-3335. doi: 10.3390/ijms15023319

Gao, F., Zhou, Y., Zhu, W., Li, X., Fan, L., and Zhang, G. (2009). Proteomic analysis of cold stress-responsive proteins in Thellungiella rosette leaves. Planta 230, 1033-1046. doi: 10.1007/s00425-009-1003-6

Geisler, M., Kleczkowski, L. A., and Karpinski, S. (2006). A universal algorithm for genome-wide in silicio identification of biologically significant gene promoter putative cis-regulatory-elements; identification of new elements for reactive oxygen species and sucrose signaling in Arabidopsis. Plant J. 45, 384-398. doi: 10.1111/j.1365-313X.2005.02634.x

Gong, Q., Li, P., Ma, S., Indu Rupassara, S., and Bohnert, H. J. (2005). Salinity stress adaptation competence in the extremophile Thellungiella halophila in comparison with its relative Arabidopsis thaliana. Plant J. 44, 826-839. doi: 10.1111/j.1365-313X.2005.02587.x

Hopkins, J., and Tudhope, G. R. (1973). Glutathione peroxidase in human red cells in health and disease. Br. J. Haematol. 25, 563-575. doi: 10.1111/j.13652141.1973.tb01768.x

Hou, Q., and Bartels, D. (2015). Comparative study of the aldehyde dehydrogenase (ALDH). gene superfamily in the glycophyte Arabidopsis thaliana and Eutrema halophytes. Ann. Bot. 115, 465-479. doi: 10.1093/aob/mcu152

Inan, G., Zhang, Q., Li, P., Wang, Z., Cao, Z., Zhang, H., et al. (2004). Salt cress. A halophyte and cryophyte Arabidopsis relative model system and its applicability to molecular genetic analyses of growth and development of extremophiles. Plant Physiol. 135, 1718-1737. doi: 10.1104/pp.104.041723

Ismail, A., Seo, M., Takebayashi, Y., Kamiya, Y., Eiche, E., and Nick, P. (2014). Salt adaptation requires suppression of jasmonate signaling. Protoplasma 251, 881-898. doi: 10.1007/s00709-013-0591-y

Jajić, I., Sarna, T., Szewczyk, G., and Strzałka, K. (2015). Changes in production of reactive oxygen species in illuminated thylakoids isolated during development and senescence of barley. J. Plant Physiol. 184, 49-56. doi: 10.1016/j.jplph.2015.06.009

Jiang, Y., Yang, B., Harris, N. S., and Deyholos, M. K. (2007). Comparative proteomic analysis of $\mathrm{NaCl}$ stress-responsive proteins in Arabidopsis roots. J. Exp. Bot. 58, 3591-3607. doi: 10.1093/jxb/erm207 
Kozieradzka-Kiszkurno, M., and Płachno, B. J. (2012). Are there symplastic connections between the endosperm and embryo in some angiosperms?-a lesson from the Crassulaceae family. Protoplasma 249, 1081-1089. doi: 10.1007/s00709-011-0352-8

Laloi, C., Stachowiak, M., Pers-Kamczyc, E., Warzych, E., Murgia, I., and Apel, K. (2007). Cross-talk between singlet oxygen-and hydrogen peroxide-dependent signaling of stress responses in Arabidopsis thaliana. Proc. Natl. Acad. Sci. U.S.A. 104, 672-677. doi: 10.1073/pnas.0609063103

Li, P., Mane, S. P., Sioson, A. A., Robinet, C. V., Heath, L. S., Bohnert, H. J., et al. (2006). Effects of chronic ozone exposure on gene expression in Arabidopsis thaliana ecotypes and in Thellungiella halophila. Plant Cell Environ. 29, 854868. doi: 10.1111/j.1365-3040.2005.01465.x

Libik-Konieczny, M., Kozieradzka-Kiszkurno, M., Desel, C., Michalec-Warzecha, $\dot{Z}$, Miszalski, Z., and Konieczny, R. (2015). The localization of NADPH oxidase and reactive oxygen species in in vitro-cultured Mesembryanthemum crystallinum L. hypocotyls discloses their differing roles in rhizogenesis. Protoplasma 252, 477-487. doi: 10.1007/s00709-014-0692-2

Lichtenthaler, H. K., and Buschmann, C. (2001). "Chlorophylls and carotenoids: measurement and characterization by UV-VIS spectroscopy," in Current Protocols in Food Analytical Chemistry, eds R. E. Wrolstad, T. E. Acree, H. An, E. A. Decker, M. H. Penner, D. S. Reid, et al. (New York, NY: John Wiley and Sons, Inc), F4.3.1-F4.3.8.

Miao, Y., Lv, D., Wang, P., Wang, X. C., Chen, J., Miao, C., et al. (2006). An Arabidopsis glutathione peroxidase functions as both a redox transducer and a scavenger in abscisic acid and drought stress responses. Plant Cell 18, 2749-2766. doi: 10.1105/tpc.106.044230

M'rah, S., Ouerghi, Z., Berthomieu, C., Havaux, M., Jungas, C., Hajji, M., et al. (2006). Effects of $\mathrm{NaCl}$ on the growth, ion accumulation and photosynthetic parameters of Thellungiella halophila. J. Plant Physiol. 163, 1022-1031. doi: 10.1016/j.jplph.2005.07.015

M'rah, S., Ouerghi, Z., Eymery, F., Rey, P., Hajji, M., Grignon, C., et al. (2007). Efficiency of biochemical protection against toxic effects of accumulated salt differentiates Thellungiella halophila from Arabidopsis thaliana. J. Plant Physiol. 164, 375-384. doi: 10.1016/j.jplph.2006.07.013

Mubarakshina, M. M., Ivanov, B. N., Naydov, I. A., Hillier, W., Badger, M. R., and Krieger-Liszkay, A. (2010). Production and diffusion of chloroplastic $\mathrm{H} 2 \mathrm{O} 2$ and its implication to signalling. J. Exp. Bot. 61, 3577-3587. doi: 10.1093/jxb/erq171

Nakano, Y., and Asada, K. (1981). Hydrogen peroxide is scavenged by ascorbatespecific peroxidase in spinach chloroplasts. Plant Cell Physiol. 22, 867-880.

Niewiadomska, E., Bilger, W., Gruca, M., Mulisch, M., Miszalski, Z., and Krupinska, K. (2011). CAM-related changes in chloroplastic metabolism of Mesembryanthemum crystallinum L. Planta 233, 275-285. doi: 10.1007/s00425010-1302-y

Niewiadomska, E., Polzien, L., Desel, C., Rozpadek, P., Miszalski, Z., and Krupinska, K. (2009). Spatial patterns of senescence and developmentdependent distribution of reactive oxygen species in tobacco (Nicotiana tabacum) leaves. J. Plant Physiol. 166, 1057-1068. doi: 10.1016/j.jplph.2008. 12.014

Niewiadomska, E., and Wiciarz, M. (2015). "Adaptations of chloroplastic metabolism in halophytic plants", in Progress in Botany, eds U. Lüttge and W. Beyschlag (Basel: Springer), 177-193.

op den Camp, R. G., Przybyla, D., Ochsenbein, C., Laloi, C., Kim, C., Danon, A., et al. (2003). Rapid induction of distinct stress responses after the release of singlet oxygen in Arabidopsis. Plant Cell 15, 2320-2332. doi: 10.1105/tpc.014662

Pang, Q., Chen, S., Dai, S., Chen, Y., Wang, Y., and Yan, X. (2010). Comparative proteomics of salt tolerance in Arabidopsis thaliana and Thellungiella halophila. J. Proteome Res. 9, 2584-2599. doi: 10.1021/pr100034f

Pang, Q., Guo, J., Chen, S., Chen, Y., Zhang, L., Fei, M., et al. (2012). Effect of salt treatment on the glucosinolate-myrosinase system in Thellungiella salsuginea. Plant Soil 355, 363-374. doi: 10.1007/s11104-011-1108-0

Pedras, M. S. C., and Adio, A. M. (2008). Phytoalexins and phytoanticipins from the wild crucifers Thellungiella halophila and Arabidopsis thaliana: rapalexin A, wasalexins and camalexin. Phytochemistry 69, 889-893. doi: 10.1016/j.phytochem.2007.10.032

Pottosin, I., and Shabala, S. (2016). Transport across chloroplast membranes: optimizing photosynthesis for adverse environmental conditions. Mol. Plant 9, 356-370. doi: 10.1016/j.molp.2015.10.006

Rastogi, A., Yadav, D. K., Szymańska, R., Kruk, J., Sedlářová, M., and Pospišil, P. (2014). Singlet oxygen scavenging activity of tocopherol and plastochromanol in Arabidopsis thaliana: relevance to photooxidative stress. Plant Cell Environ. 37, 392-401. doi: 10.1111/pce.12161

Sewelam, N., Jaspert, N., Van Der Kelen, K., Tognetti, V. B., Schmitz, J., Frerigmann, H., et al. (2014). Spatial H2O2 signaling specificity: H2O2 from chloroplasts and peroxisomes modulates the plant transcriptome differentially. Mol. Plant 7, 1191-1210. doi: 10.1093/mp/ssu070

Stepien, P., and Johnson, G. N. (2009). Contrasting responses of photosynthesis to salt stress in the glycophyte Arabidopsis and the halophyte Thellungiella: role of the plastid terminal oxidase as an alternative electron sink. Plant Physiol. 149, 1154-1165. doi: 10.1104/pp.108.132407

Suzuki, N., Koussevitzky, S., Mittler, R., and Miller, G. (2012). ROS and redox signalling in the response of plants to abiotic stress. Plant Cell Environ. 35, 259-270. doi: 10.1111/j.1365-3040.2011.02336.x

Taji, T., Seki, M., Satou, M., Sakurai, T., Kobayashi, M., Ishiyama, K., et al. (2004). Comparative genomics in salt tolerance between Arabidopsis and Arabidopsisrelated halophyte salt cress using Arabidopsis microarray. Plant Physiol. 135, 1697-1709. doi: 10.1104/pp.104.039909

Tiwari, A., Rác, M., and Pospíšil, P. (2013). Formation of superoxide anion and carbon-centered radicals by photosystem II under high light and heat stress-EPR spin-trapping study. J. Bioenerg. Biomembr. 45, 551-559. doi: 10.1007/s10863-013-9523-y

Uzilday, B., Ozgur, R., Sekmen, A. H., Yildiztugay, E., and Turkan, I. (2015). Changes in the alternative electron sinks and antioxidant defence in chloroplasts of the extreme halophyte Eutrema parvulum (Thellungiella parvula) under salinity. Ann. Bot. 115, 449-463. doi: 10.1093/aob/mcu184

Wiciarz, M., Gubernator, B., Kruk, J., and Niewiadomska, E. (2015). Enhanced chloroplastic generation of $\mathrm{H} 2 \mathrm{O} 2$ in stress-resistant Thellungiella salsuginea in comparison to Arabidopsis thaliana. Physiol. Plant. 153, 467-476. doi: $10.1111 /$ ppl.12248

Wiciarz, M., Niewiadomska, E., and Kruk, J. (2016). Different effects of salt stress on low molecular antioxidants and redox state of photosynthetic electron carriers of Eutrema salsugineum (halophyte) and Arabidopsis thaliana (glycophyte). Acta Physiol. Plant. (in press).

Wong, C. E., Li, Y., Labbe, A., Guevara, D., Nuin, P., Whitty, B., et al. (2006) Transcriptional profiling implicates novel interactions between abiotic stress and hormonal responses in Thellungiella, a close relative of Arabidopsis. Plant Physiol. 140, 1437-1450. doi: 10.1104/pp.105.070508

Wu, H. J., Zhang, Z., Wang, J. Y., Oh, D. H., Dassanayake, M., Liu, B., et al. (2012). Insights into salt tolerance from the genome of Thellungiella salsuginea. Proc. Natl. Acad. Sci. U.S.A. 109, 12219-12224. doi: 10.1073/pnas.1209954109

Yamane, K., Taniguchi, M., and Miyake, H. (2012). Salinity-induced subcellular accumulation of $\mathrm{H}_{2} \mathrm{O}_{2}$ in leaves of rice. Protoplasma 249, 301-308. doi: 10.1007/s00709-011-0280-7

Conflict of Interest Statement: The authors declare that the research was conducted in the absence of any commercial or financial relationships that could be construed as a potential conflict of interest.

Copyright (c) 2016 Pilarska, Wiciarz, Jajić, Kozieradzka-Kiszkurno, Dobrev, Vanková and Niewiadomska. This is an open-access article distributed under the terms of the Creative Commons Attribution License (CC BY). The use, distribution or reproduction in other forums is permitted, provided the original author(s) or licensor are credited and that the original publication in this journal is cited, in accordance with accepted academic practice. No use, distribution or reproduction is permitted which does not comply with these terms. 